\title{
What contributes to quality of life in patients with Parkinson's disease?
}

\author{
Anette Schrag, Marjan Jahanshahi, Niall Quinn
}

\begin{abstract}
Objective-To identify the factors that determine quality of life (QoL) in patients with idiopathic Parkinson's disease in a population based sample. Quality of life (QoL) is increasingly recognised as a critical measure in health care as it incorporates the patients' own perspective of their health.

Methods-All patients with Parkinson's disease seen in a population based study on the prevalence of parkinsonism were asked to complete a disease-specific QoL questionnaire (PDQ-39) and the Beck depression inventory. A structured questionnaire interview and a complete neurological examination, including the Hoehn and Yahr scale, the Schwab and England disability scale, the motor part of the unified Parkinson's disease rating scale (UPDRS part III), and the mini mental state examination were performed by a neurologist on the same day.
\end{abstract}

Results-The response rate was $78 \%$. The factor most closely associated with QoL was the presence of depression, but disability, as measured by the Schwab and England scale, postural instability, and cognitive impairment additionally contributed to poor QoL. Although the UPDRS part III correlated significantly with QoL scores, it did not contribute substantially to predicting their variance once depression, disability, and postural instability had been taken into account. In addition, patients with akinetic rigid Parkinson's disease had worse QoL scores than those with tremor dominant disease, mainly due to impairment of axial features.

Conclusion-Depression, disability, postural instability, and cognitive impairment have the greatest influence on QoL in Parkinson's disease. The improvement of these features should therefore become an important target in the treatment of the disease.

(F Neurol Neurosurg Psychiatry 2000;69:308-312)

Neurology, Institute of

Neurology, Queen

Square, London

WC1N 3BG, UK

A Schrag

M Jahanshahi

N Quinn

Correspondence to:

Professor NP Quin

n.quinn@ion.ucl.ac.uk

Received 2 Sepyember 1999 and in revised form

10 February 2000

Accepted 24 February 2000 instrument for use with patients with Parkinson's disease, the PDQ-39, has been shown to have good reliability, validity, responsiveness, and reproducibility, ${ }^{2-4}$ and is now used in many trials to assess effectiveness of treatment. ${ }^{5}$ Its disease specificity and the single summary index offer the opportunity to assess the overall impact of illness, and it is easy to interpret. ${ }^{6} \mathrm{We}$ used this instrument to determine which disease related factors, such as presence of specific symptoms, degree of impairment, disability, depression, or cognitive impairment, are the most relevant for quality of life in a population based sample of patients with Parkinson's disease.

Patients and methods

All patients with Parkinson's disease seen in a population based study on the prevalence of parkinsonism were included. The methods of case ascertainment and diagnosis have been described in detail elsewhere. ${ }^{7}$ Briefly, the records of 15 general practices in the area of London covering a population of 121608 were screened for patients with a suspicion of Parkinson's disease or parkinsonism, for patients with tremor with onset after the age of 50, and for patients who had ever received antiparkinsonian drugs. As the health care system in the United Kingdom is organised locally the population of a general practice is representative of the surrounding area. In addition, the general practitioner acts as a "gatekeeper" of the Health Service, so that patients are only referred to specialists by their general practitioners. Patients who agreed to participate had a complete neurological examination and a structured questionnaire interview which were performed by a movement disorders specialist (AS). Parkinson's disease was diagnosed according to the United Kingdom brain bank criteria $^{8}$ with the exception that patients with an isolated Babinski's sign but otherwise typical Parkinson's disease were not excluded. Patients with atypical parkinsonian disorders, drug induced parkinsonism, vascular pseudoparkinsonism, and those with parkinsonism after dementia were excluded from this study. The assessment included the Hoehn and Yahr scale, ${ }^{9}$ the Schwab and England disability scale, ${ }^{10}$ the motor part of the unified Parkinson's disease rating scale (UPDRS) ${ }^{11}$ and the mini mental state examination (MMSE). ${ }^{12}$ In addition, patients were questioned about the presence or absence of symptoms and signs associated with Parkinson's disease, such as hallucinations, falls, gait impairment, motor fluctuations or dyskinesias, incontinence, orthostatic symptoms, insomnia, pain, speech, or swallowing impairment, or a family history of 
Table 1 Patient characteristics $(n=92)$

\begin{tabular}{lll}
\hline & $n$ & $\%$ \\
\hline Hoehn and Yahr stage: & 11 & 12.0 \\
I & 8 & 8.7 \\
I.5 & 22 & 23.9 \\
II & 28 & 30.4 \\
II.5 & 17 & 18.5 \\
III & 2 & 2.2 \\
IV & 4 & 4.3 \\
V & $72.0(10.9)$ & \\
Mean age (y) & $5.3(4.1)$ & \\
Mean disease duration (y) & $49: 43$ & \\
Sex (M:F) & $66.7(12.0)$ & \\
Mean age of onset (y) & $23.4(12.1)$ & \\
Mean UPDRS score & $12.0(10.5)$ & \\
Mean BDI score & $27.3(4.0)$ & \\
Mean mini mental state score & & \\
\hline
\end{tabular}

Values in parentheses are SD.

Parkinson's disease. They were also asked which single symptom had been predominant at onset and whether they were unemployed or had retired early due to having Parkinson's disease. At the time of the diagnostic visit, all patients were also given the PDQ-39, a disease specific, 39 item questionnaire on QoL in Parkinson's disease, ${ }^{2}$ the Beck depression inventory (BDI), ${ }^{13}$ as well as two other QoL instruments (EQ-5D and short form-36) ) $^{14}$ which are not the subject of this paper, and asked to complete and return them within the next 2 weeks in a stamped, addressed envelope.

INSTRUMENTS

The PDQ-39 comprises 39 questions, each with five different answer options (never, occasionally, sometimes, often, or always). Eight subscores (mobility, activities of daily living, emotional wellbeing, stigma, social support, cognition, communication, and bodily discomfort), and one summary index, can be calculated (maximum score 100 indicating worst level of problem). The BDI is a widely used 21 item depression inventory with answer options from $0-3$ and a maximum score of 63 . The cut off score for moderate to severe depression used was 17 (18 or more indicates moderate to severe depression). ${ }^{16}$ Cognitive impairment was measured using the mini mental examination scale ${ }^{12}$ with a cut off score of 24 or less.

\section{SCORING AND STATISTICAL ANALYSIS}

Summary scores as well as the subdimensions of the PDQ-39 were calculated according to the scoring algorithm. ${ }^{17}$ The akinesia score of the UPDRS was calculated as the sum of item 19 and 23 to 26 of the UPDRS for both sides, the score of axial features from items 27 to 30, and the tremor score from items 20 and 21 for

Table 2 Clinical features associated with significantly impaired quality of life scores

\begin{tabular}{lllr}
\hline & \multicolumn{2}{l}{ Mean PDQ-39 summary index scores } & \\
\cline { 2 - 4 } & $\begin{array}{l}\text { Feature present } \\
(S D)\end{array}$ & $\begin{array}{l}\text { Feature not present } \\
(S D)\end{array}$ & p Value \\
\hline Depression (BDI>17); $\mathrm{n}=18$ & $39(18.3)$ & $16.7(11.2)$ & $<0.001$ \\
MMSE<25; $\mathrm{n}=13$ & $49.4(19.2)$ & $24.4(15.9)$ & $<0.001$ \\
History of hallucinations ${ }^{\star} ; \mathrm{n}=15$ & $39(16.2)$ & $27.2(19)$ & $0.026 \dagger$ \\
History of falls $; \mathrm{n}=58$ & $36.2(18.4)$ & $21.4(17.1)$ & $<0.001$ \\
Postural instability; $\mathrm{n}=43$ & $33.7(19.3)$ & $18.7(11.8)$ & $<0.001$ \\
Gait impairment ${ }^{\star} ; \mathrm{n}=61$ & $35.6(19.1)$ & $20(15)$ & $<0.001$ \\
Akinetic-rigid subtype; $\mathrm{n}=68$ & $20.7(2.8)$ & $14.6(3.3) \ddagger$ & 0.005 \\
\hline
\end{tabular}

*Patient report on interview; tonly marginally significant; łtremor dominant subtype; BDI=Beck depression inventory score; MMSE=mini mental state examination score. both sides. The tremor dominant subtype of Parkinson's disease was defined as patients with a ratio of tremor to bradykinesia score (bradykinesia rigidity and postural instability subscores from the UPDRS motor scale and history) of 0.5 or more, and the akinetic rigid subtype as patients with a ratio of<0.5. Socioeconomic groups were determined according to the classification of occupations and coding index with socioeconomic group 1 representing the highest and group 5 the lowest socioeconomic class. ${ }^{18}$

Mean values were compared by the MannWhitney test, and Spearman rank correlation coefficients were calculated to assess the direction and magnitude of association between variables. Stepwise multiple regression analysis was used to determine the factors that best accounted for variance in QoL scores. Due to the number of different comparisons, statistical significance was only accepted at $\mathrm{p}<0.005$.

\section{Results}

Among all patients seen $(n=202), 124$ were classified as having probable Parkinson's disease. The response rate was $78 \%(97 / 124$ questionnaires). There was no significant difference between responders and nonresponders for age, disease duration, sex, Hoehn and Yahr stage, and Schwab and England score $(p>0.05)$. Five questionnaires were returned with incomplete data $(<50 \%$ of the QoL instrument), and were not used for further analysis. The characteristics of the responders are given in table 1 .

\section{IMPACT OF CLINICAL FEATURES ON PDQ-39} SCORES

Patients with high levels of depression (with BDI scores $>17$ ), a mini mental state score of 24 or less (out of 30 ), ${ }^{12}$ postural instability on examination (as described in the UPDRS), and a history of falls or of gait difficulties had significantly worse PDQ-39 summary index scores than patients without these features (table 2). QoL scores of patients with the akinetic rigid subtype of Parkinson's disease were also worse than those with tremor dominant disease. This difference could not be explained by older age or longer disease duration, as age and disease duration were similar between those with tremor dominant and those with akinetic rigid Parkinson's disease. The difference of QoL scores between those with and those without a history of hallucinations just failed to reach significance $(p=0.026$; table 2). No difference in PDQ-39 scores was found between men and women and between patients with or without a poor (subjectively $<50 \%$ ) initial or current response to antiparkinsonian medication. A history of dyskinesias or fluctuations, incontinence, orthostatic symptoms, insomnia, pain, speech or swallowing impairment, a family history of Parkinson's disease, and symptom at onset had no significant impact on QoL scores. There was also no difference between those who were unemployed or had retired early due to the disease and those who were not, those with disease 
Table 3 Results of stepwise multiple regression analysis of PDQ-39 scores

\begin{tabular}{|c|c|c|c|c|c|c|}
\hline Predictors & $\begin{array}{l}\text { Standardised } \\
\text { regression } \\
\text { coefficient }\end{array}$ & $R^{2}$ & $R^{2}$ change & $t$ Value & $p$ Value & \\
\hline Beck depression inventory score & 0.47 & & 0.54 & 5.45 & $<0.0001$ & \\
\hline Schwab and England disability score & -0.31 & 0.64 & 0.1 & 3.41 & $<0.001$ & \\
\hline Postural instability & 0.24 & 0.69 & 0.05 & 3.34 & $<0.001$ & \\
\hline \multirow[t]{3}{*}{ Mini mental state examination score } & -0.17 & 0.72 & 0.02 & 2.14 & $<0.037$ & \\
\hline & & & & & Multiple & $\begin{array}{l}R=0.85 \\
R^{2}=0.72\end{array}$ \\
\hline & & & & & Adjusted & $R^{2}=0.70$ \\
\hline
\end{tabular}

$\star$ Regression coefficient for each individual variable.

$\mathrm{R}^{2}$ is the proportion of the PDQ 39 variation explained by the model taking into account the listed factors cumulatively.

onset before or after the age of 50, and those with current age older than 60 or 70 .

CORRELATIONS OF CLINICAL SCORES WITH PDQ-39 The PDQ-39 summary index correlated significantly and positively $(r=0.68, \mathrm{p}<0.001)$ with depression as measured by the BDI score and negatively with disability as measured by the Schwab and England scale $(r=-0.66$, $\mathrm{p}<0.001$ ). Significant correlations were also obtained with disease severity as measured by the Hoehn and Yahr scale $(r=0.6, \mathrm{p}<0.001)$, the UPDRS motor subscore of axial features $(r=0.57, \mathrm{p}<0.001)$, the total UPDRS motor score $(\mathrm{r}=0.41, \mathrm{p}<0.001)$, and the MMSE $(r=-0.32, \mathrm{p}<0.001)$. Correlations with duration of disease and age were not significant $(r=0.18, \mathrm{p}=0.19$ and $r=0.14, \mathrm{p}=0.25$, respectively), and akinesia score $(r=0.3, \mathrm{p}=0.028)$ and socioeconomic groups $(r=0.25, \mathrm{p}=0.023)$ just failed to correlate significantly with QoL.

REGRESSION ANALYSIS OF PDQ-39 SCORES

To determine which factors contributed most to QoL scores, we performed a stepwise linear regression, entering age, disease duration, and all factors that were shown to have an impact on QoL scores (see previous sections) into the regression in a stepwise fashion (table 3 ). The most important predictive factor was the BDI depression score, followed by disability as measured by the Schwab and England score, which together accounted for $64 \%$ of the variance of QoL scores. Additional contributing factors were postural instability and cognitive impairment as measured by the MMSE score, which accounted for a further $8 \%$ of the variance of QoL scores. To account for some of the overlap of contents in quality of life and disability measures, as well as the Schwab and England with Hoehn and Yahr scores $(r=-0.74, \mathrm{p}<0.0001)$, we performed a separate analysis excluding Schwab and England scores from the analysis. Depression, Hoehn and Yahr, and MMSE scores then accounted for $59 \%$ of the variance of quality of life scores.

\section{Discussion}

In this study, the strongest predictor of QoL in Parkinson's disease was the presence of depression, as measured by the Beck depression inventory score. This correlation of QoL with depression has also been found in other studies on QoL in Parkinson's disease, accounting for up to $60 \%$ of impairment of PDQ-39 scores, ${ }^{19}{ }^{20}$ but also in other chronic diseases where depression has been found to have a major influence on QoL scores. ${ }^{21}$ It could be argued that the PDQ-39 and the BDI measure the same content and hence have a strong correlation. However, the PDQ-39 comprises eight different dimensions of which depression features in only one. In addition, the results were similar when the two other administered, generic, QoL instruments (EQ-5D and short form-36) were used. The results of the EQ-5D will be reported in detail elsewhere. Using the same variables in the regression analysis together with a history of hallucinations, $64 \%$ of the variance of the generic QoL measure EQ-5D was possible. When a regression analysis was performed on the physical summary score of the short form-36, 33\% of its variance was predicted by the Schwab and England score and postural instability alone, whereas $23 \%$ of the variance of the mental summary score of the SF-36 was predicted by the BDI alone. No other factors contributed to prediction of the variance in these two generic QoL scores. When considering the results of all these regression analyses, it should be noted that the direction of causality between QoL, depression, and disability is difficult to establish with correlational methods. Nevertheless, the association of Parkinson's disease with depression is well known, but treatment of depression in the disease is often insufficient. ${ }^{22}$ The results of this study highlight the need to diagnose and treat depression in Parkinson's disease more effectively to improve the patients' QoL.

As expected, QoL was also strongly related to disability as measured by the Schwab and England scores. However, there is some overlap in the content of QoL and disability scales, and, additionally, disability scores also strongly correlated with disease severity, as measured by the Hoehn and Yahr scale $(r=-0.74$, $\mathrm{p}<0.0001)$. When two variables are very strongly associated - for example, with correlations in the order of 0.8 or 0.9 - collinearity is a potential source of error in regression analysis. Although the correlation between the measures of disability and disease severity was only 0.74 , we nevertheless performed a separate regression analysis excluding disability as a variable. The results of this analysis were similar but, as might be expected, disease severity replaced disability as a predictor of QoL. In addition, postural instability, which is one of the key criteria for staging in the Hoehn and Yahr scale and was therefore associated with higher Hoehn and Yahr scores $(p<0.005)$, no longer additionally contributed to predicting 
QoL scores. Depression and MMSE scores, on the other hand, continued to contribute to the prediction of QoL scores. Scores on the MMSE were not strongly associated with depression $(r=-0.15$, NS) or Hoehn and Yahr scores $(r=-0.27, \mathrm{p}<0.05)$. As cognitive impairment does not respond to, and can be aggravated by, medication that improves mobility, it presents a major problem in the management of Parkinson's disease, and has been shown to be the primary reason for nursing home placement. ${ }^{23}$ Our results further confirm the importance of cognitive impairment in determining QoL.

The correlation of QoL scores with measures of disease severity and disability has been shown previously ${ }^{2} 2024$ and is not surprising. However, the motor part of the UPDRS, which is the primary outcome measure for most treatment trials in Parkinson's disease, correlated less strongly with QoL scores. This scale, by contrast with the Hoehn and Yahr scale, involves a detailed assessment of parkinsonian features, comprising items of speech, hypomimia, tremor, and rigidity in addition to bradykinesia and axial features such as postural instability and gait. However, these subscores did not have a significant influence on QoL scores. As a consequence, the Hoehn and Yahr scale, which reflects predominantly axial features, correlated more strongly with QoL scores than the motor part of the UPDRS. This suggests that the Hoehn and Yahr scale may be a more sensitive measure than the motor part of the UPDRS to evaluate the impact that severity of disease has on the patients' everyday life.

Tremor and rigidity scores did not correlate with QoL scores. Gait problems and limb akinesia, on the other hand, were associated with worse QoL, although not contributing further to its prediction once disease severity was accounted for. Similarly, Peto et $a l^{2}$ found that self reported slowness was significantly related to seven out of the eight subdimensions of the PDQ-39, whereas self reported tremor and rigidity were much less markedly correlated with the PDQ-39 subdimensions. We also found that patients with the akinetic rigid subtype of PD (which incorporates greater impairment of axial features) had worse QoL scores than those with the tremor dominant subtype of Parkinson's disease. In studies assessing subtypes of parkinsonism, tremor dominant disease has usually been found to have a better prognosis than the akinetic rigid subtype, with a slower rate of progression of motor symptoms. ${ }^{25}{ }^{26}$ Our findings of a better QoL in tremor dominant Parkinson's disease further support this differentiation. Symptoms at onset, on the other hand, did not significantly affect QoL scores. However, information on type of symptom at onset was collected by patient report, which has a low reliability. ${ }^{27}$

A history of hallucinations on antiparkinsonian treatment just failed to be significantly associated with worse QoL scores, and a history of fluctuations of motor response to medication or presence of dyskinesias were also not associated with poor QoL. However, these results may be due to the relatively few patients with these complications which, if present, were usually not severe in this population based sample.

In accordance with our findings, Karlsen et $a l^{24}$ also found that depression and disability are important predictors of QoL in Parkinson's disease. However, by contrast with our study, postural instability did not correlate as strongly with QoL, whereas insomnia, which did not predict QoL in our study, was an important influential factor in their study. This difference is likely to arise from their use of the Nottingham Health Profile (NHP), which is a generic rather than a disease specific instrument, and therefore does not address specific problems that arise in Parkinson's disease. It has been used to assess QoL in patients with Parkinson's disease in a large study of Sinemet CR, ${ }^{28}$ but its validity in patients with Parkinson's disease has not been formally tested. In addition, it is highly skewed at the severe end of ill health, and will not detect subtle impairments. The section on sleep is larger in the NHP than in the disease specific PDQ-39, and insomnia is therefore more likely to correlate with NHP scores. The disease specific PDQ-39 used in this study, on the other hand, is based on problems reported by patients with Parkinson's disease $^{2}$ and some correlations of this instrument with symptoms of Parkinson's disease therefore result from its development. Our findings are, however, enhanced by the result that when two other, generic, QoL instruments were used, the same factors were predictive of the variance of QoL scores. The prediction of variance of QoL scores was similar when the EQ-5D was used as a dependent variable, whereas it was lower when the separate physical and mental summary scores of the SF-36 were used. The SF-36 has been shown also in other disorders to have a low sensitivity to discriminate between patient differences in overall QoL. ${ }^{29}$ Although high correlations of PDQ-39 scores may be partly due to the design of this disease specific instrument, the similar albeit less significant results with the two generic instruments therefore support the validity of our findings.

The QoL is a complex concept to which many factors other than health contribute. This complexity of the concept of QoL is also the main limitation of this study, as many variables that potentially contribute to QoL, such as social support and individual coping strategies, were not directly measured. Also, we have no information on variables such as the cultural context of the patients' lives or availability of healthcare resources, which may have great potential for modifying the impact of the disease on the QoL in Parkinson's disease. However, lower socioeconomic group just failed to be significantly associated with worse QoL scores.

On the positive side, we think that the results of this study can be generalised to a wider population of patients with Parkinson's disease, as the patients were seen in a population based epidemiological study with a high response rate $(84 \%)$. Also, the results are clinically relevant 
in this patient group due to the disease specificity of the instrument used.

We conclude that QoL in idiopathic Parkinson's disease is primarily determined by depression, disability, disease severity (particularly related to axial features), and cognitive impairment. Patients with the akinetic rigid subtype have worse QoL scores than those with tremor dominant disease. The motor part of the UPDRS, although the primary outcome measure for clinical trials with antiparkinsonian medication, did not contribute significantly to QoL in Parkinson's disease, and additional emphasis on other disease features should therefore gain greater importance in the treatment of the disease.

This study was made possible by a grant from SmithKline Beecham Pharmaceuticals. MJ is funded by the Wellcome Trust.

1 Lang AE, Lozano AM. Parkinson's disease. Second of two parts. N Engl f Med 1998;339:1130-43.

2 Peto V, Jenkinson C, Fitzpatrick R, et al. The development and validation of a short measure of functioning and well being for individuals with Parkinson's disease. Qual Life Res 1995;4:241-8.

3 Peto V, Jenkinson C, Fitzpatrick R. PDQ-39: a review of the development, validation and application of a Parkinson's disease quality of life questionnaire and its associated measures. $\mathcal{f}$ Neurol 1998;245(suppl 1):S10-4.

4 Fitzpatrick R, Peto V, Jenkinson C, et al. Health-related quality of life in Parkinson's disease: a study of outpatien clinic attenders. Mov Disord 1997;12:916-22.

5 Fitzpatrick R, Jenkinson C, Peto V, et al. Desirable properties for instruments assessing quality of life: evidence from the PDQ-39. F Neurol Neurosurg Psychiatry 1997;62: 104 .

6 Jenkinson C, Fitzpatrick R, Peto V, et al. The Parkinson's disease questionnaire (PDQ-39): development and validation of a Parkinson's disease summary index score. Age Ageing 1997;26:353-7.

7 Schrag A, Ben-Shlomo Y, Quinn NP. The population prevalence of atypical parkinsonian disorders. Lancet 1999;354: lence of

8 Gibb WR, Lees AJ. The relevance of the Lewy body to the pathogenesis of idiopathic Parkinson's disease. If Neurol Neurosurg Psychiatry 1988;51:745-52.

9 Hoehn MM, Yahr MD. Parkinsonism: onset, progression and mortality. Neurology 1967;17:427-42.

10 Schwab RS, England AC. Projection technique for evaluating surgery in Parkinson's disease. In: Gillingham FJ, Donaldson IML, eds. Third Symposium on Surgery in Parkinson' disease. Edinburgh: Livingstone, 1969:152-7.

11 Fahn S, Elton RL, members of the UPDRS Development Committee. Unified Parkinson's disease rating scale. In: Fahn S, Marsden CD, Calne DB, et al, eds. Recent developments in Parkinson's disease. Vol 2. Florham Park, NJ: MacMillan Healthcare Information, 1987:153-63.
12 Folstein MF, Folstein SE, McHugh PR. Mini-mental state. A practical method for grading the cognitive state of patients for the clinician. F Psychiatr Res 1975;12:189-98.

13 Beck AT, Ward C, Mendelson M, et al. An inventory for measuring depression. Arch Gen Psychiatry 1961;4:561-7.

14 EuroQoL Group. EuroQoL: a new facility for the measurement of health-related quality of life. Health Policy 1990;16: 199-208.

15 McHorney CA, Ware JE Jr, Raczek AE. The MOS 36-item short-form health survey (SF-36): II. Psychometric and clinical tests of validity in measuring physical and mental health constructs. Med Care 1993;31:247-63.

16 Jahanshahi $M$, Marsden CD. Depression in torticollis: a controlled study. Psychol Med 1988;18:925-33.

17 Jenkinson C, Fitzpatrick R, Peto V. The Parkinson's disease questionnaire. User manual for the $P D Q-39, P D Q-8$ and $P D Q$ summary index. Oxford: Joshua Horgan Print Partnership, 1998.

18 Office of Population Censuses and Surveys. Classification of occupations and coding index, 1980. London: The Stationery Office, 1980.

19 Martinez-Martin P, Frades Payo B. Quality of life in Parkinson's disease: validation study of the PDQ-39 Spanish version. The Grupo Centro for Study of Movement Disorders. 7 Neurol 1998; (suppl 1):S34-8.

20 Findley L, Global Parkinson's Disease Steering Committee. Investigating factors which may influence quality of life in Parkinson's disease. Parkinsonism and Related Disorders 1999;5(suppl):146.

21 King RB. Quality of life after stroke. Stroke 1996;27:1467-

22 Livingston G, Watkin V, Milne B, et al. The natural history of depression and the anxiety disorders in older people: the Islington community study. F Affect Disord 1997;46:25562.

23 Goetz CG, Stebbins GT. Risk factors for nursing home placement in advanced Parkinson's disease. Neurology 1993;43:2227-9.

24 Karlsen KH, Larsen JP, Tandberg E, et al. Influence of clinical and demographic variables on quality of life in patients with Parkinson's disease. I Neurol Neurosurg Psychiatry 1999;66:431-5.

25 Zetusky WJ, Jankovic J, Pirozzolo FJ. The heterogeneity of Parkinson's disease: clinical and prognostic implications. Neurology 1985;35:522-6.

26 Jankovic J, McDermott $M$, Carter J, et al. Variable expression of Parkinson's disease: a base-line analysis of the DATATOP cohort. The Parkinson Study Group. Neurology 1990;40:1529-34.

27 Richards M, Marder K, Cote L, et al. Reliability of symptom onset assessment in Parkinson's disease. Mov Disord 1994; 9:340-2.

28 Block G, Liss C, Reines S, et al. Comparison of immediaterelease and controlled release carbidopa/levodopa in Parkinson's disease. A multicenter 5-year study. The CR First Study Group. Eur Neurol 1997;37:23-7.

29 Williams LS, Weinberger M, Harris LE, et al. Measuring quality of life in a way that is meaningful to stroke patients. Neurology 1999;53:1839-43.

30 Brazier JE, Walters SJ, Nicholl JP, et al. Using the SF-36 and Euroqol on an elderly population. Qual Life Res 1996;5: 195-204. 